University of Nebraska - Lincoln

DigitalCommons@University of Nebraska - Lincoln

$11-22-2004$

\title{
Simulation of ultrashort electron pulse generation from optical injection into wake-field plasma waves
}

D.S. Dodd

University of Michigan - Ann Arbor

J.K. Kim

University of Michigan - Ann Arbor

Donald P. Umstadter

University of Nebraska-Lincoln, donald.umstadter@unl.edu

Follow this and additional works at: https://digitalcommons.unl.edu/physicsumstadter

Part of the Physics Commons

Dodd, D.S.; Kim, J.K.; and Umstadter, Donald P., "Simulation of ultrashort electron pulse generation from optical injection into wake-field plasma waves" (2004). Donald Umstadter Publications. 9.

https://digitalcommons.unl.edu/physicsumstadter/9

This Article is brought to you for free and open access by the Research Papers in Physics and Astronomy at DigitalCommons@University of Nebraska - Lincoln. It has been accepted for inclusion in Donald Umstadter Publications by an authorized administrator of DigitalCommons@University of Nebraska - Lincoln. 


\title{
Simulation of ultrashort electron pulse generation from optical injection into wake-field plasma waves
}

\author{
E. S. Dodd, * J. K. Kim, and D. Umstadter \\ Center for Ultrafast Optical Science, University of Michigan, Ann Arbor, Michigan 48109, USA \\ (Received 19 December 2003; revised manuscript received 13 July 2004; published 22 November 2004)
}

\begin{abstract}
A laser-plasma-based source of relativistic electrons is described in detail, and analyzed in two dimensions using theoretical and numeric techniques. Two laser beams are focused in a plasma, one exciting a wake-field electron plasma wave while another locally alters some electron trajectories in such a way that they can be trapped and accelerated by the wave. Previous analyses dealt only with one-dimensional models. In this paper two-dimensional particle-in-cell simulations and analysis of single particle trajectories show that the radial wake field plays an important role. The simulation results are interpreted to evaluate the accelerated electron beam's properties and compared with existing devices.
\end{abstract}

DOI: 10.1103/PhysRevE.70.056410

PACS number(s): 52.38.Kd, 41.75.Jv, 52.65.Rr, 29.25.Bx

\section{INTRODUCTION}

Nearly 20 years ago, the use of laser-induced plasma waves to accelerate charged particles was proposed [1]. These wake-field accelerators seek to take advantage of the ultra-high electron acceleration gradients $(>10 \mathrm{GeV} / \mathrm{m})$ of laser-driven plasma waves [2-4], which are made possible due to the invention of compact, high-peak-power lasers [5]. The plasma-wave electric field gradients are three orders of magnitude higher than those in conventional rf linacs, because they are not limited by dielectric breakdown. Normally, electrons oscillating in the plasma wave cannot be accelerated by the wake field since they are out of phase with it. Electrons that are not part of the plasma wave can become trapped, or continuously accelerated by the wave, provided that they are moving in the correct phase at nearly the phase velocity of the wave [6]. Since this velocity is close to the speed of light, it was generally thought that such preacceleration can only be accomplished by external injection, such as with a conventional linac. However, the low-field gradient $(<10 \mathrm{MeV} / \mathrm{m})$ [7] of a first-stage conventional linac prolongs the time during which beam emittance can grow before the beam becomes relativistic; after this point, self-generated magnetic fields can balance the effects of space charge. Gradients on the order of $1 \mathrm{GeV} / \mathrm{cm}$ have been demonstrated experimentally [8], and accelerate electron beams with transverse emittances that rival current electron guns.

Acceleration of electrons by plasma waves requires first a means to drive the wave, and second a method for electron injection. In the former case, when an intense laser pulse propagates through a plasma, its ponderomotive force displaces plasma electrons. Since this force is proportional to the laser intensity gradient, it will be directed primarily in the longitudinal direction, if the laser pulse length is much shorter than its focal spot size. Because the ions remain stationary, due to their larger inertia, a residual charge imbalance results after the laser pulse passes by. Thus, a large

*Present address: Los Alamos National Laboratory, Los Alamos, New Mexico 87545, USA. amplitude plasma wave will be resonantly driven behind the laser pulse if the pulse width approximately equals a plasmawave period. The resulting electrostatic wake field propagates in the same direction, with a phase velocity equal to the group velocity of the laser pulse. This wake becomes the gradient for particle acceleration. In terms of a method for electron injection, even with state-of-the-art electron guns, the pulse width of the electron bunch can be considerably longer than the plasma wave period of a second-stage laserplasma accelerator. It will thus fill multiple acceleration phases uniformly, resulting in a large energy spread. Also, it is difficult to position and focus the electron beam in the plasma channel with micrometer accuracy, and synchronize it with the plasma wave acceleration phase.

In previous work, we showed that all these problems could simply be solved by making use of an additional laser pulse $[9,10]$. This method also takes advantage of the excellent emittance properties of plasma-based electron generation, while providing an easily built experimental setup [11]. The basic idea is that once a wake field is excited by the longitudinal ponderomotive force of one laser pulse (the pump pulse), the ponderomotive force of a second laser pulse (the injection pulse) can then be used to locally alter the trajectories of the plasma wave electrons such that they are in phase with the wave's electric field and accelerated to the trapping velocity. The key is that electrons are dephased, either directly by the laser pulse or by other means, which will be discussed. As first described in a previous paper [9], this device is referred to as laser injected laser accelerator, or LILAC.

Since initial publication of the idea, a number of variations on optical injection have been proposed [12]. However, none of these dealt with transverse effects of the plasma waves, which can be significant, as pointed out in [13]. One paper which did was [14]; however, their results show much higher transverse emittance than the work presented here. To properly understand the physics of the device, the analysis was reworked in this paper to include transverse effects. These calculations are then compared to the simulations to show that two-dimensional effects are essential. With the multidimensional simulations, the emittance, or quality of 


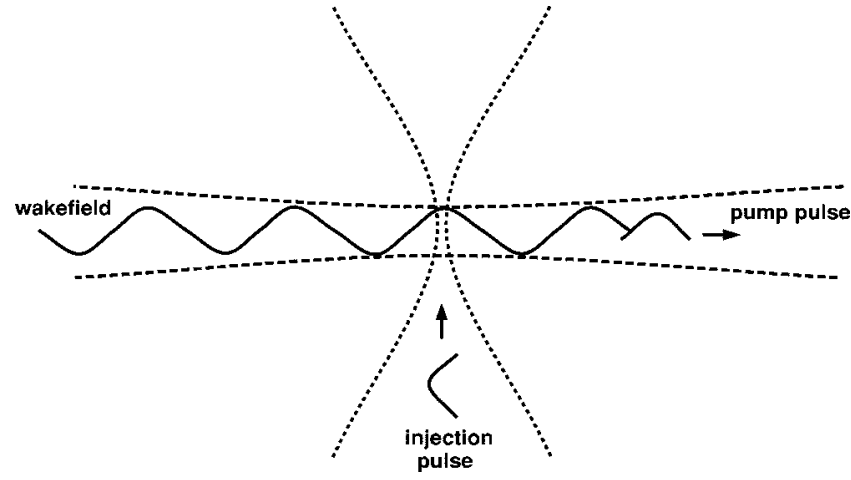

FIG. 1. Schematic of the crossed-laser-plasma wave accelerator concept.

the beam, can be evaluated, along with the effect of the radial component to the wake field. Both numeric and theoretical methods were used to evaluate the performance and possible uses for LILAC, as compared with conventional accelerators.

This paper is organized as follows. In Sec. II, a simple analysis is used to obtain an approximate expression for the intensity threshold of the injection pulse in order to trap electrons. We next present in Sec. III, the results from a more realistic analysis of the motion of a single electron in the combined wake field and the injection pulse. In Sec. IV, we present the results of two-dimensional (2D) particle-in-cell (PIC) code simulations, which include collective effects, such as the perturbation caused by the wake of the injection pulse, yielding details about the accelerated electron beam, such as its pulse duration, emittances, current, and energy spread. We conclude in Sec. V.

\section{MODEL}

The LILAC concept consists mainly of three different stages. First, a large amplitude wake field is generated; second, electrons are dephased; and then third, electrons are trapped in the pump's wake field from the dephasing. By understanding each of the three parts involved, a description of the injection process can be derived. The process of dephasing and then trapping electrons with secondary laser pulses is quite general. Just as there are myriad ways to drive plasma waves, by means of either a single pulse, multiple pulses, or beat waves, there are many ways to use lasers to inject electrons. Several orientations of the laser pulses are also possible: collinear, counterpropagating, or orthogonal. Besides combining laser pulse characteristics, ionization or density gradients are other possible means to dephase electrons. This paper is useful in two respects: first, in detailing one example of a laser-based electron gun, and second, by providing an example framework for future work. In the analysis that follows, the model will be used to study the specific case of orthogonal geometry, shown in Fig. 1 and discussed in the previous paper. The pump pulse and its wake will be examined first, since they are independent of the version of all-optical injection.

\section{A. Background theory}

Laser-plasma based accelerators utilize the electrostatic field of a plasma wave to accelerate electrons, for which there exist three main generation techniques. The laser wakefield accelerator (LWFA) [1-4] uses a single pump pulse with a pulse width $\tau_{p} \sim 2 \pi / \omega_{p}$, where $\omega_{p}$ is the plasma frequency. Another technique, the beat-wave accelerator, uses the beat of two longer pulses to generate a train of short pulses [15]. Finally, the most efficient method is the resonant laser-plasma accelerator [16], which uses a series of pump pulses with increasing spacing between them and decreasing pulse widths to compensate for the change in resonance as the plasma wave grows and $\omega_{p}$ changes. Although any pumping method is compatible with LILAC, for the sake of simplicity, we will primarily consider the LWFA.

In order for electrons to be accelerated, they must become trapped in the plasma wave. When electrons begin to move with the wave, its potential appears to be a well, instead of oscillatory; at this point trapping becomes similar to the Kepler problem. There exists a minimum energy above which the particle is trapped and accelerated, and below which it oscillates in the background. The intensity of the pump pulse defines both the accelerating gradient and the threshold for trapping. The pump laser pulse is described by $\mathbf{a}=\mathbf{a}_{\mathrm{env}}(z, t) e^{i \omega_{0} t}=e \mathbf{A}_{\perp} / m_{e} c^{2}$, where $\mathbf{A}_{\perp}$ is the transverse vector potential and $\omega_{0}$ is the frequency of the pump. Denoting $\Phi$ and $v_{g}$ as the electrostatic potential and the group velocity of the wake, respectively, the $1 \mathrm{D}$ governing equation for the wake field, characterized by a plasma frequency $\omega_{p}$, is given by

$$
\frac{d^{2} \phi}{d \zeta^{2}}=k_{p}^{2} \gamma_{g}^{2}\left[\beta_{g}\left(1-\frac{1+a^{2}}{\gamma_{g}^{2}\left(1+\phi^{2}\right)}\right)^{-1 / 2}-1\right],
$$

where $\quad \zeta=z-c t, \quad \phi=e \Phi / m_{e} c^{2}, \quad \beta_{g}=v_{g} / c \approx \beta_{\phi}, \quad \gamma_{g}$ $=\left(1-\beta_{g}^{2}\right)^{-1 / 2} \approx \gamma_{\phi} \approx \omega_{0} / \omega_{p}$, and the plasma wave number $k_{p}$ $=\omega_{p} / c[17]$. Given a properly optimized pump pulse width, the largest normalized amplitude for the electric field of a plasma wave, given a, is $E_{\max }$ given in [17]. The background electrons experience an electric field of the wake which is $\pi / 2$ out of phase with their momentum in the plasma, and thus none are trapped. However, if the amplitude of the plasma wave's electric field is very large, a small amount of dephasing of the electron momentum with respect to the field can result in trapping and acceleration of these electrons.

\section{B. Longitudinal trapping}

Now that the wake is defined, trapping may be described for a given amplitude. For a particle's longitudinal motion this has already been derived in at least two different papers [6]. Both papers define a threshold in the electron energy, above which electrons move with the wave and are accelerated. This threshold is defined in phase space by an orbit called the separatrix, because it separates the region of closed, trapped orbits from open ones. The separatrix is obtained by equating the kinetic energy of electron with the wave's potential energy in the wave frame, and then transforming back to the lab frame. The threshold injection energy of electrons is given for any value of $\phi$, at phase $\zeta$, if $\phi$ is a function of $\zeta$. Thus, from [6],

$$
\Gamma=\gamma_{\phi}\left(1+\gamma_{\phi} \epsilon\right) \pm \gamma_{\phi} \beta_{\phi}\left\{\left(1+\gamma_{\phi} \epsilon\right)^{2}-1\right\}^{1 / 2},
$$




$$
\epsilon=\phi(\zeta)-\phi\left(\zeta_{0}\right)
$$

where $\Gamma$ is the relativistic gamma factor for the electrons kinetic energy. The separatrix results when $\zeta_{0}$ is the phase for the minimum potential. If the phase of the maximum potential, $\phi_{\max }-\phi_{\min }$, is substituted into $\epsilon$ then Eq. (2) gives the minimum trapping energy. Thus for a given pump pulse, Eq. (1) yields the amplitude of the wave, and Eq. (2) gives the kinetic energy an electron needs to be trapped.

\section{Transverse trapping}

Equation (2) is limited to one dimension, since it uses only the longitudinal velocity. Electrons will also have transverse momentum, causing them to drift out of the wake to one side, unless some sort of focusing, or transverse trapping, guides them. However, the potential also has a transverse dimension and as the particle moves transversely in the wake potential $\phi(\zeta, r)$, it can be turned back towards the axis, if the field is strong enough. To stay trapped we assume the particle oscillates about the axis $(r=0)$ and reaches a maximum radial position $(r=r)$ where it turns back. At these two points we define $\phi\left(\zeta_{0}, 0\right)=0$ on axis, and $\phi\left(\zeta_{0}, r\right)=\Delta \phi$ at the maximum radial position. On axis, the electron has its maximum transverse momentum, $p_{y}$, besides the necessary longitudinal momentum for trapping, $p_{z 1}$, for a total initial energy of $\gamma_{i}=\sqrt{p_{y}^{2}+p_{z 1}^{2}+1}$, where $\Gamma_{1}=\sqrt{p_{z 1}^{2}+1}$ is calculated from Eq. (2). At the maximum radius of a particle's transverse motion the transverse component of its momentum is $p_{y}=0$, and the longitudinal trapping momentum at this point is $p_{z 2}$. This gives a final total energy $\gamma_{f}=\sqrt{p_{z 2}^{2}+1}$ $=\Gamma_{2}$ that is just equal to the minimum longitudinal trapping energy, at $r=r$. From basic physics, the change in kinetic energy is equal and opposite to the change in potential energy, $\Delta \phi=-\Delta \gamma$. Therefore, the equation to be solved is

$$
\gamma_{f}=\gamma_{i}-\Delta \phi \text {. }
$$

Substituting in the initial and final energies, we arrive at the final form

$$
p_{y}^{2}(r=0)=\Delta \phi\left(2 \Gamma_{2}+\Delta \phi\right)+\left(\Gamma_{2}^{2}-\Gamma_{1}^{2}\right) .
$$

The maximum transverse momentum on axis, $p_{y}$, that can remain trapped in the wave is related to the depth of the potential well $\Delta \phi$, and the change in minimum longitudinal trapping as the electron moves off axis, $\Gamma_{1}$ and $\Gamma_{2}$. When $\Delta \phi, \Gamma_{1}$, and $\Gamma_{2}$ are measured from a PIC code, Eq. (5) gives a condition for transverse motion in a wake that Eq. (2) had previously given only for the longitudinal case. One may estimate $p_{y}$ from the potential in simulation results, an example of which is plotted in the inset of Fig. 8, which will be discussed in Sec. IV B.

As an example assume a wave with phase velocity $\gamma_{\phi}$ $=10$ and wave amplitude of $\epsilon=0.7$ on axis, then from Eq. (2) $\Gamma_{1}=1.0243$ and $p_{z 1}=0.22$. As the particle drifts off axis it reaches some maximum radius, $3 \mu \mathrm{m}$ for example, before turning back toward the center. At this point let the amplitude have decreased to $\epsilon=0.5$ so that $\Gamma_{2}=1.1350$ and $p_{z 2}=0.54$. If the potential difference between this point and the axis is $\Delta \phi=0.3$ then the maximum transverse momentum on axis

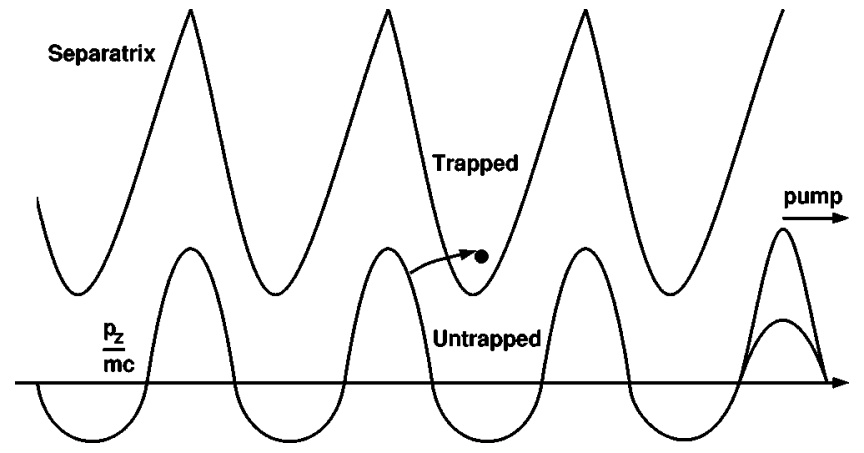

FIG. 2. Basic profile for LWFA. The pump pulse creates a plasma wave to accelerate electrons. To be accelerated, electrons must cross inside the separatrix.

that can remain within the $3 \mu \mathrm{m}$ radius without escaping is $p_{y}=1.0$ from Eq. (5). This equation will be used in Sec. IV to evaluate the amount of focusing needed for LILAC, at which point simulation data will be used. It should be pointed out here that the same calculation was performed independently in [14], with the result in a different form.

\section{Injection mechanisms}

The next step is to discuss ways to move electrons from oscillating in the background across the separatrix and thus accelerate them to any desired energy, Fig. 2. Previous discussions of laser based accelerators assumed that an electron bunch would be injected from an external gun, however this has the drawbacks mentioned in Sec. I. The oscillations of electrons in large amplitude plasma waves take them very close to the separatrix without becoming trapped, such that only a small impulse is needed to make them cross it. Dephasing may arise from a number of sources, such as density variations, ionization, interaction between multiple waves, and the ponderomotive force of additional laser pulses. If the density varies, so will $\omega_{p}$, causing neighboring electrons to oscillate at different frequencies. This form of dephasing will be mentioned again in Sec. IV due to its presence in simulations. In ionization, newly freed electrons may appear at velocities different from older electrons at the same phase, i.e., dephased. Also, the wave may interact with other wake-fields, and the ponderomotive potentials of other laser pulses, causing complicated orbits. These orbits may cross the original separatrix, mixing phase space in any case. The new orbits may be calculated if the combined potentials are known. This situation has been studied for the specific case of a wake field overlapped with the ponderomotive potential of a second pulse [18]. The results show orbits that connect background oscillation to accelerated forward motion for the duration of the second, or injection pulse. Regardless of method, any possible injection scheme involves Eqs. (1), (2), and (5). Having selected a trapping method, the dephasing and effect on the wake field may be calculated analytically or numerically. The specific case which follows can be treated as an example of how to approach any injection scheme.

We assume that electrons interact with the injection pulse through the ponderomotive force, the same force used in 
deriving Eq. (1). The pulse will dephase electrons via this potential, distorting the plasma wave and separatrix, causing some electrons to enter the separatrix and become trapped, Fig. 3. The phase space area with trapped electrons defines the emittance of the beam, and is shaded in the figure. Emittance will be discussed in Sec. IV. It is now necessary to calculate the ponderomotive force due to the injection pulse in order to describe trapping. In the specific case of transversely oriented pulses, the injection pulse is given a Gaussian temporal and spatial profile, described by the pulse width $\tau$, and the beam radius at the focal point $r_{0}$, as well as the amplitude $b_{0}$. The normalized vector potential for the injection pulse, $\mathbf{b}$, can then be written as

$$
\mathbf{b}=\mathbf{b}_{\mathrm{env}}(r, t) e^{i \omega t},
$$

where $\mathbf{b}_{\text {env }}(r, t)=\mathbf{b}_{0} \exp \left[-\left(r / r_{0}\right)^{2}\right] \exp \left\{-4\left[\left(t-t_{c}\right) / \tau\right]^{2}\right\}$ is the pulse profile, $t_{c}$ is the time the peak of the injection pulse crosses the $z$ axis, and $\omega$ is the laser frequency. Here $b_{0}$ takes the place of $a_{0}$ to distinguish the pump from injection pulse, however they have the same meaning. The focal point of the injection pulse is at $z=0$ and $y=0$. In this specific case, $r$ of the injection pulse is in the $z$ direction. The time averaged relativistic ponderomotive force of the injection pulse is defined by [19]

$$
\mathbf{F}_{\text {pond }}=-\frac{1}{\left(1+\mathbf{b}^{2}\right)^{1 / 2}} \frac{m_{e} c^{2}}{4} \nabla \mathbf{b}^{2} .
$$

Reference [19] and the following calculation assume that any plasma response to the injection pulse can be neglected. We feel this is reasonable since the conclusions of this paper are based on: one, numerical solutions to single particle motion that give similar results in Sec. III; and two, PIC simulation results that contain the full plasma response in Sec. IV. The results of this section are meant as a guide for the correct order of magnitude. A more complete calculation would need a ponderomotive force containing the full plasma response, and Ref. [20] contains such a calculation, though for a different problem. The ponderomotive force, Eq. (7), is in both the positive and negative $z$ directions, producing bidirectional drift motion of the electrons in the plasma wave. Those electrons that acquire a ponderomotive drift velocity

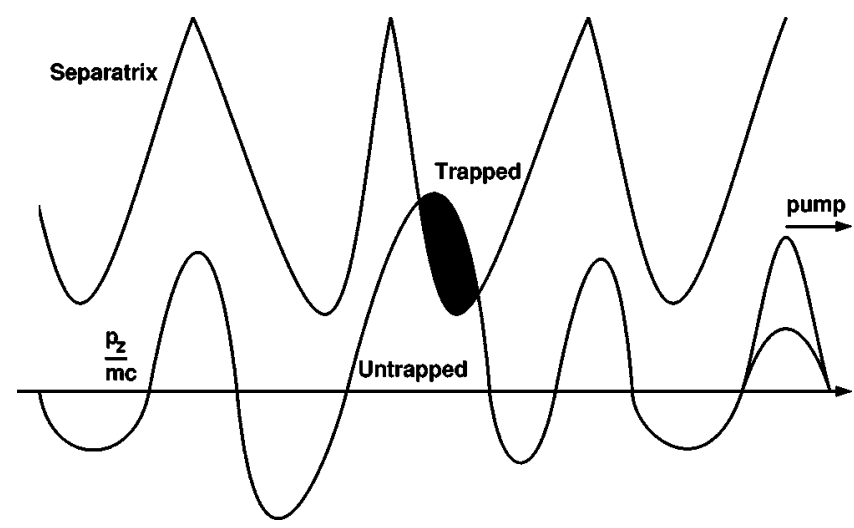

FIG. 3. Basic profile for optical injection. The injection pulse dephases electrons in the plasma wave, distorting it and the separatrix. Electrons cross the separatrix, into the accelerating bucket. in the same direction as the pump pulse, are velocity dephased with respect to, and injected into, the wake field, and will be trapped if inside the separatrix. The local perturbation of the injection pulse can also disrupt the phase of the wake field, disabling LILAC operation in the third stage. However, the propagation velocity of this disturbance is much less than the phase velocity of the plasma wave. This means that once electrons are injected into the plasma wave, the disturbance will not affect the ongoing acceleration.

A condition for trapping based on the intensities of the pump and injection pulses is necessary. In what follows, we have developed an analytic theory which provides a simple, intuitive model for trapping in one dimension. An electron receives a drift velocity from the transverse ponderomotive force of a laser pulse. If this velocity exceeds the calculated threshold at an injection point in the plasma wave, then the electron will be trapped. We first start with the relativistic ponderomotive force exerted on an electron, undergoing a series of plasma oscillations initiated by the pump. Plugging Eq. (6) into Eq. (7), we get

$$
\begin{aligned}
\left|\mathbf{F}_{\text {pond }}\right|= & \frac{b_{0}^{2}}{\left(1+b_{0}^{2} / 2\right)^{2}} \frac{m_{e} c^{2}}{r_{0}^{2}}\left[z \exp \left(-2 \frac{z^{2}}{r_{0}^{2}}\right)\right] \\
& \times\left[\exp \left(-8 \frac{\left(t-t_{c}\right)^{2}}{\tau^{2}}\right)\right] .
\end{aligned}
$$

Since, for a propagating pulse, $z$ depends on $t$, in order to determine the ponderomotive force, we should solve the equation of motion and find $z(t)$. The equation of motion for electron's trajectory is

$$
\frac{d}{d t}\left(\gamma m_{e} \mathbf{v}\right)=q \mathbf{E}_{\mathrm{epw}}+\mathbf{F}_{\mathrm{pond}}
$$

where $\mathbf{E}_{\text {epw }}$ is the electric field of the plasma wave in the $z$ direction. Noting that the scheme relies on alteration of the electron velocity distribution, and hence alters the wake field, solving this problem exactly is nontrivial. To avoid this difficulty, we first solve the system with the assumption that the wake field is a function of $\zeta$ only, meaning that the ponderomotive force does not affect the wake, in the simplest case. Further we assume that the electron drifts a negligible distance during dephasing, so that we need not compute $z(t)$. Thus it is straightforward to get an approximate functional form for the change of the dimensionless relativistic momentum $\Delta(\gamma \beta)$. Using an electron whose plasma oscillation center is $r_{0} / 2$ away from $z=0$ along the positive $z$ axis, the ponderomotive force assumes positive maximum value. For a beam diameter of the injection pulse equal to the plasma wavelength $2 r_{0}=\lambda_{p}=\omega_{p e} c$, the injection pulse produces

$$
\begin{gathered}
\Delta(\gamma \beta)=\Delta(\gamma \beta)_{0} \delta\left(t-t_{c}\right), \\
\Delta(\gamma \beta)_{0}=\frac{b_{0}^{2}}{\left(1+b_{0}^{2} / 2\right)^{1 / 2}} \sqrt{\frac{\pi}{8}} \exp (-1 / 2) .
\end{gathered}
$$

Equation (11) represents the impulse an electron receives by drifting out of the injection pulse, with the $\delta$ function being a short impulsive kick. Alone this equation only describes test electrons with no space charge, or interaction with the wake. 
With an idea of which electrons are being dephased, the proper combination of trapping threshold and injection pulse intensity will lead to the desired trapping threshold.

\section{E. Injection threshold}

In order to calculate the injection point in the plasma wave, it is first necessary to define the background plasmawave electron motion. We can simply write down the nonlinear oscillatory motion of the electron as follows:

$$
\begin{gathered}
\gamma \beta=-(\gamma \beta)_{0} p(t), \\
z=z_{0} p\left(t+\pi / \omega_{p}\right), \\
\phi=\phi_{0} p(t-z / c),
\end{gathered}
$$

where $(\gamma \beta)_{0}$ and $z_{0}$ refer to the amplitude of the motion, and $p(t)$ represents the form of the plasma wave. For a lowamplitude linear wave $p(t)$ can have a sine or cosine shape. In the nonlinear regime it takes a form that is solved for numerically [16]; nevertheless, it is always a periodic function. Due to the $\delta$ function in Eq. (11), the momentum of the electron experiences a discontinuity at $t=t_{c}$ and is dephased with respect to other background plasma wave electrons,

$$
(\gamma \beta)\left(t_{c}\right)=-(\gamma \beta)_{0} p\left(t_{c}\right)+\Delta(\gamma \beta)_{0} .
$$

Combining Eq. (11) for the dephasing with Eq. (2) for the minimum trapping velocity, we can get $b_{t h}$, the threshold value for $b_{0}$, which is required in order to trap the electron for a given $\phi\left(a_{0}\right)$,

$$
b_{t h}=\frac{S}{2}\left[1+\left(1+16 / S^{2}\right)^{1 / 2}\right]^{1 / 2},
$$

where

$$
S=\sqrt{\frac{8}{\pi}} \exp (1 / 2)\left[\left(\Gamma^{2}-1\right)^{1 / 2}+(\gamma \beta)_{0} p\left(t_{c}\right)\right] .
$$

For example, with $\gamma_{\phi}=10$ and $a_{0}=1$, which corresponds to $\epsilon=0.7$, Eq. (16) predicts $b_{t h} \approx 0.8$. Even though this model illustrates the essential physics of the LILAC, it is a simplified description because the injection of electrons is assumed to occur at the maximum of $\phi$ where the injection is optimal, i.e., perfect phase matching. Thus it reveals little information about the threshold's dependence on the temporal separation between the injection and pump pulses, described by $t_{c}$. Yet $t_{c}$ significantly affects $b_{t h}$ since it provides the synchronization between the accelerating phase of the electric field of the plasma wave and the ponderomotive push by the injection pulse.

As we will see in the following sections, it suffices to predict the needed order of magnitude of the laser pulse intensities. A more complicated version must be solved for numerically. Also, since this same process may be followed for any given laser injection configuration, dephasing can be compared with the trapping threshold to determine if injection will occur. In our simple model we have added the drift velocity from the injection pulse to a single electron, how- ever we can think of it in more general terms: the change to the wake's potential is essentially equal to the original wake plus the ponderomotive potential. So in this section we have provided a trapping threshold for a specific injection scheme, while trying to use a general prescription for analyzing the dephasing of electrons due to a second laser pulse. In order to test the concept fully, the threshold will be analyzed via simulation in the following sections.

\section{ANALYSIS OF MOTION OF A SINGLE ELECTRON}

The concept is first tested by studying the relativistic equation of motion for a single electron. The test particle moves within the plasma wave's two dimensional electric field governed by the numerical integration of Eq. (9). The electron is allowed to move in the $y-z$ plane to properly describe the interaction between the electron and the injection pulse. Thus Eq. (9) is decomposed into the $y$ and $z$ components:

$$
\begin{gathered}
\frac{d}{d t}\left(\gamma m_{e} \beta_{y}\right)=\frac{q}{c} \beta_{z} E_{\mathrm{inj}}, \\
\frac{d}{d t}\left(\gamma m_{e} \beta_{z}\right)=\frac{q}{c}\left\{\left(1-\beta_{y}\right) E_{\mathrm{inj}}+E_{\mathrm{epw}}\right\} .
\end{gathered}
$$

We assume the following: the group velocity of the wake $v_{g}$ is approximately equal to $c$ and is a constant; the quasistatic approximation; a linear polarization for the injection pulse along the $z$ axis; no wake is produced by the injection pulse; and the plasma channel is one dimensional. The plasma wake field is generated by solving one-dimensional fluid equations numerically and imported to $\mathbf{E}_{\text {epw }}$.

First, we verified the validity of the code by comparing it with analytic expressions for (1) the final drift velocity and (2) the threshold trapping energy. For the calculation of the final drift velocity, good agreements are made when $b_{0}$ is smaller than 1.3; the deviation starts to occur due to the fact that the amplitude of the ponderomotive force in the curve is always calculated at $z=r_{0} / 2$. With large $b_{0}$, the displacement $z(t)$ of the electron becomes important and the assumption of maximum ponderomotive force no longer holds. Trapping threshold of electrons in the plasma wave agrees with Eq. (2) for any $\epsilon$. After the validity check, simulations with both pump and injection pulse were then performed. Even though this analysis cannot provide us with information about the number and energy spread of the accelerated electrons, it is still useful to determine the optimal time delay between the pump and the injection pulse as well as the threshold intensity for the injection pulse when the amplitude for the pump pulse is given.

In order to follow the motion of an electron in the plasma wave, we first assume the plasma channel is one dimensional in the $z$ direction. This can be justified by using a spot size for the pump much larger than for the injection pulse. Thus, the transit time in which the injection pulse crosses the channel is much larger than a plasma period $\left(\tau_{\text {transit }} \gg \tau_{p}\right)$. Furthermore, the plasma wave is assumed to be free of Landau damping and any perturbation by the injection pulse. In 

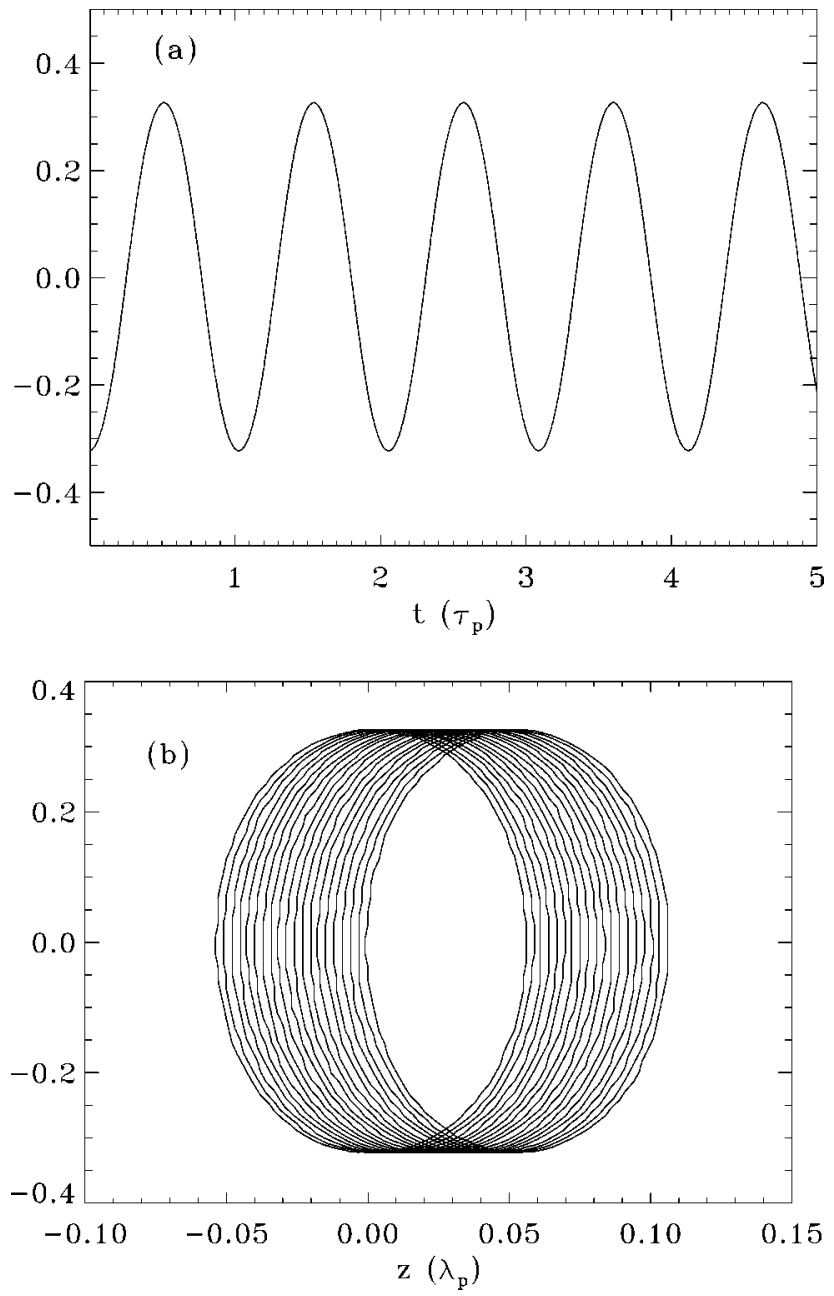

FIG. 4. A particle oscillating in the background plasma wave. (a) is $\beta_{z}$ vs $t$. (b) is $\beta_{z}$ vs $z$. Note the Stokes drift in (b).

short, the pump solely determines the behavior of the plasma wave.

A single electron, with initial conditions for velocity and position consistent with other background electrons in the plasma wave, is allowed to undergo plasma oscillations along the $z$ axis. Since the excursion distance is on the order of $\lambda_{p} / 2 \pi$ without injection, the motion can be approximated by a harmonic oscillator system. In Fig. 4, the motion of this background electron is shown, from a pump pulse with $a_{0}$ $=1$ having generated the plasma wave. The ratio between the laser frequency and the plasma frequency was 10:1, the same as the relativistic factor for the plasma wave, $\gamma_{\phi}$. The phase space trajectory in Fig. 4(b) is that of a harmonic oscillator, including the Stokes drift of the particle due to the wave's finite phase velocity [21].

The electron trajectory drastically changes when the injection pulse is applied. The peak of the injection pulse arrives at $z=0$ on the $z$ axis at time $t=t_{c}$. Again, the pump and injection pulse laser frequencies were equal. A value of $t_{c}$ $=2.5 \tau_{p}$ was used, corresponding to the negative maximum of the potential. Also $a_{0}=1$ and $b_{0}=1.7$ were used respectively for the pump and injection pulse. As seen in Fig. 5(a), the electron was clearly injected into the plasma wave, but be-
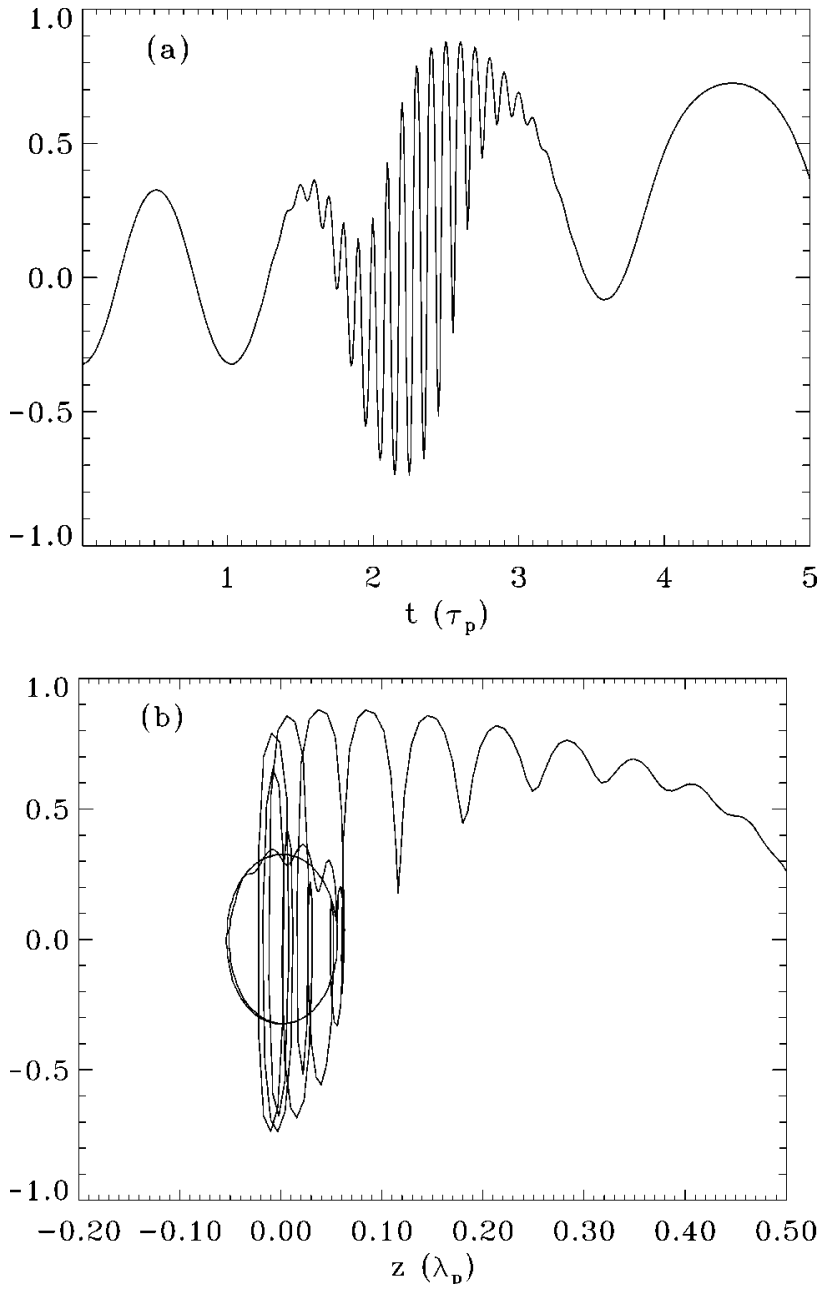

FIG. 5. A particle oscillating in the background with injection. The injection pulse changes the motion of the electron. The phase is incorrect and the particle is not trapped in the wave. (a) is $\beta_{z} \mathrm{vs} t$. (b) is $\beta_{z}$ vs $z$.

cause of the poor synchronization between the plasma wave field and the ponderomotive force from the injection pulse, the electron could not obtain the required thrust from the plasma wave field to remain in the same "bucket" of plasma wave and thus was not trapped. Figure 5(b) shows this bucket-to-bucket transition of the electron in phase space trajectory. Thus optimization of $t_{c}$ is crucial in order to minimize $b_{0}$ for threshold operation of LILAC.

Next, keeping the other parameters fixed, the same simulation was done except with $t_{c}=1.85 \tau_{p}$, which approximately corresponds to the maximum of the potential when the peak of the injection pulse crosses the $z$ axis. Figure 6 shows that electron trapping occurred given this value of $b_{0}$. The plasma wave phase velocity was $0.995 c$ and the electron was observed to pass the trapping threshold velocity and continued to be accelerated up to $21 \mathrm{MeV}$ within $40 \tau_{p}$, corresponding to an energy gain of $50 \mathrm{keV}$ per micron. The angular distance produced by the longitudinal ponderomotive force of the injection pulse was approximately $0.025 \mathrm{rad}$ after $40 \tau_{p}$ (corresponding to a distance of $10 \mu \mathrm{m}$ off the $z$ axis along $y$ or a distance of $\lambda_{p}$ ). The ratio between the $y$ and $z$ directional velocities was $0.6 \%$. In order to obtain the 

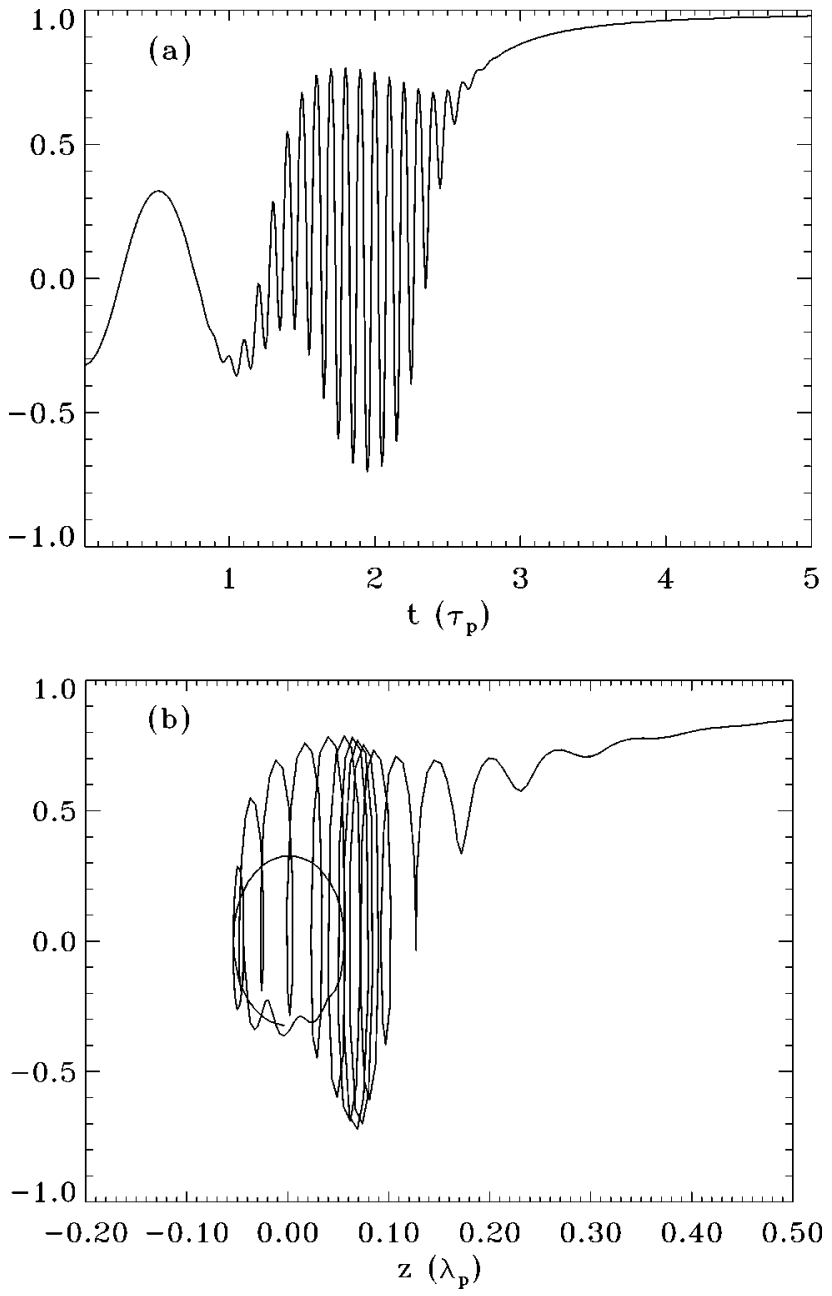

FIG. 6. (a) Velocity of an injected and trapped electron in LILAC, predicted by Eq. (9), is plotted versus time. The optimized time delay between the pump and injection pulse, $t_{c}=1.85 \tau_{p}$, was used. With the same parameters as Fig. 5 except $t_{c}$, the electron was trapped and remained in the same bucket until the end of simulation. (b) The phase space trajectory was plotted, $\beta_{z}$ vs $z$.

maximum acceleration, the electron must remain in the plasma channel in the transverse dimension. Although the electron in the above calculation did remain in a 1D plasma channel ( $r_{\text {epw }} \gg \lambda_{p}$ where $r_{\text {epw }}$ is the waist size of the plasma wave), this effect could be a limitation on the maximum acceleration. However, it can be readily removed by the use of two colliding counterpropagating injection pulses right at the center of plasma channel. Given proper temporal and spatial synchronization, their longitudinal ponderomotive drifts cancel each other, adding constructively only to a transverse ponderomotive drift. Alternatively, the radial wake can be used. To move transversely out of the wake the electron needs to overcome the trapping potential, provided the kinetic energy of the electron is large enough for escape. Therefore a finite transverse profile helps to keep the beam aligned with the axis, if the radial wake has a high enough amplitude.

To show the crucial dependence of $b_{t h}$ on $t_{c}, t_{c}$ was varied within the range of one plasma wavelength in multiple simulations. A value of $a_{0}=1$ and a value of $b_{0}=1.6$, were used to

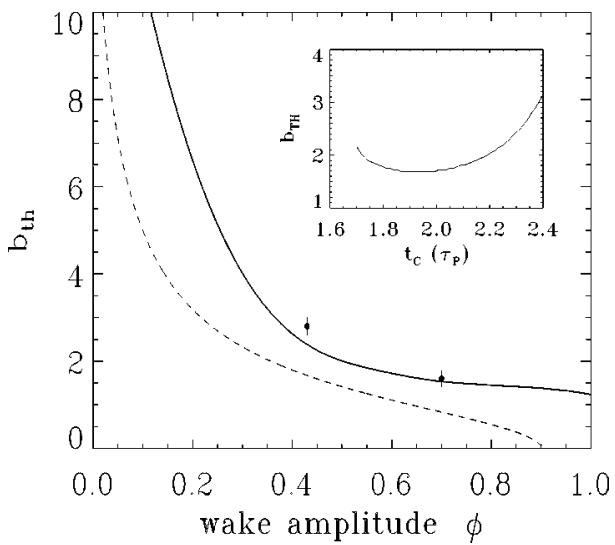

FIG. 7. The trapping threshold $b_{\text {th }}$ plotted versus the plasmawave amplitude $\phi$. The dashed line represents the results of Eq. (16) and the solid one, Eq. (9). The trapping region is above the curves. The results of PIC simulations are shown by the two points; the error bars are from finite temperature effects. Inset: $(\gamma \beta)_{z}$ vs $t_{c}$, valid only along $y=0$.

determine the optimal value for $t_{c}$ in Fig. 7 (inset). Figure 6 shows the resulting trajectory of these simulations. Because of the periodicity of the plasma wave, the optimum value for $t_{c}$ also has period $\tau_{p}$. Since the variation of $t_{c}$ in the simulation was within $\tau_{p}$, the periodicity of $t_{c}$ is implicitly assumed hereafter. The optimal value was found to be $t_{c}=1.85 \tau_{p}$. The electron was not trapped since $b_{0}=1.6$ was short of $b_{0}=1.7$, the optimized threshold value. However, it is clear that $t_{c}$ $=1.85 \tau_{p}$ is optimal with an injection pulse of $b_{0}=1.6$. Since the plasma channel has a finite waist size, $t_{c}$ is a linear function of $y$ in the channel. This implies that only near $y=0$ will a given value of $t_{c}$ be optimal.

Figure 7 compares the calculation of Sec. II and numerical simulation of Sec. III. The threshold value for $b_{0}$ is plotted versus the amplitude of the plasma wave. The solid line represents Eq. (9) with $\gamma_{\phi}=10$ and the dashed line results from Eq. (16), including consideration of $t_{c}$. For a wave with a higher phase velocity, e.g., a value of $\gamma_{\phi}=100$, this theory predicts a larger threshold $b_{t h}$, since then an electron needs a stronger boost from the injection pulse in order to be trapped in the faster plasma wave.

Note the distinct discrepancy between curves at $\phi=0.9$, where the dashed line goes to zero while the threshold points from Eq. (9) remain above $b_{0}=1.3$. In arriving at Eq. (16), optimal injection (perfect phase matching) of a test electron is implicitly assumed. Accordingly, the final drift velocity of the electron from the injection pulse is independent of the amplitude and phase of the wake. An electron with zero initial velocity injected at maximum $\phi$ can be self-trapped (without an injection pulse). On the other hand, when Eq. (9) is solved, the injected background electron has a phase difference relative to that of the wake, determined by the delay parameter $t_{c}$. Self-trapping no longer occurs (at least not in a cold plasma for wave amplitudes below wave breaking).

$b_{\text {th }}$ becomes independent of $\phi$ as $\phi$ approaches 1 because while it becomes easier to trap an electron it also becomes harder to dephase one. These two effects begin to balance each other. The electron is easier to trap because of the larger 
TABLE I. A summary of the different simulation sets used in the study of LILAC. Three sets of simulations were performed, each containing seven to ten simulations.

\begin{tabular}{ccc}
\hline \hline Set & Purpose & Varied \\
\hline 1 & Find optimum wake & $a_{0}, r_{0}, \gamma_{\phi}$ \\
2 & Test LILAC, find $b_{t h}$ & Timing between injection pulse and pump \\
3 & Test lack of focusing on trapping & Used $r_{0}=5 \mu \mathrm{m}$ for pump \\
\hline \hline
\end{tabular}

$\phi$, decreasing the required injection threshold velocity. In contrast, it becomes harder to dephase an electron oscillating in a large amplitude plasma wave because the required change in momentum becomes larger.

\section{PARTICLE-IN-CELL SIMULATIONS}

To test the principle of optical injection, including the effect of the injection pulse's wake, particle-in-cell simulations were performed. Previously, 1D simulations of LILAC were performed [9], as well as initial 2D runs [10]. In this section, the results of a two dimensional code will be detailed and compared with the previous data. In all simulations, the pump pulse has a wavelength of $1 \mu \mathrm{m}$, and a pulse length $10 \mu \mathrm{m}$, or $33 \mathrm{fs}$, giving electron densities of $\sim 10^{19} \mathrm{~cm}^{-3}$ and $\tau_{p} \sim \tau_{l}$, where $\tau_{l}$ is the laser pulse length. Therefore, the one and two dimensional simulations may be compared directly. The pump pulse used a Gaussian spatial profile with a spot size arrived through simulation, as will be discussed. The spatial domain size for the simulation was $5 \lambda_{p}$ in $2 \mathrm{D}$, using a grid of $1024 \times 1024$ cells with $5 \times 10^{6}$ particles. The PIC code, called TRISTAN [22], is based on the same relativistic and electromagnetic algorithm in both one and two dimensions. It is fully self-consistent so the vector potential satisfies, $\boldsymbol{\nabla} \cdot \mathbf{A}=0$, for Gaussian laser pulses. Two changes in the code have been made: a shifting routine was written to remain in the moving frame of the laser pulse, and the two dimensional code was moved to a parallel machine, IBM SP2, using a domain decomposition algorithm. The basic simulation code was unchanged, parallelism was added with Message Passing Interface (MPI) and all interprocessor communication was hidden inside separate routines.

\section{A. Parameters studied}

To determine the importance of two-dimensional effects and to study geometries that do not require the onedimensional approximation, three simulation sets were run (see Table I). The first varies the pump pulse's parameters, the second studies LILAC, and the third tests LILAC without transverse focusing. This paper primarily concerns itself with the results of the second set, where the two pulses start overlapped, and then the delay between them is increased. As before, we use the LWFA with the pump pulse resonant to the plasma frequency. The specific pump pulse parameters used are $a_{0}=1.6, r_{0}=8 \mu \mathrm{m}$, and $\tau_{p}=10 \lambda_{l} / c$, where $\lambda_{l}$ is the laser's wavelength. Given the spot size, the Rayleigh range was $180 \mu \mathrm{m}$ for $2 \mathrm{D}$. For the injection pulse we use $\tau=2 \tau_{p}$ and $b_{0}=2.0$ with a spot size of $r_{0}=5 \mu \mathrm{m}$. In Sec. IV B we present the electron phase space resulting from the simulations described.

The pump parameters detailed above resulted from the first set of simulations. The various spot sizes of the pump were $r_{0}=5,6.5$, and $8 \mu \mathrm{m}$; the intensity was varied from $a_{0}=1.6$ to 2.2 ; and the pulse length was 16.7 or $33 \mathrm{fs}$, giving phase velocities of $\gamma_{\phi}=5$ and 10 . The previously published 2D simulations used $\gamma_{\phi}=5$ [10]; however, background trapping obscured the trapping threshold, though injection was observed. Therefore background reduction was needed. For this reason, the phase velocity of the wave was raised to $\gamma_{\phi}=10$, thus the need for such a large simulation grid. In the case of $a_{0} \geqslant 2$, there was some self trapping of electrons from the background, leading to the choice of $a_{0}=1.6$. Also as discussed in the model section, transverse trapping, or focusing of the electrons is necessary in order for trapping to occur, therefore the spot size was varied to find the minimum required: $r_{0}=8 \mu \mathrm{m}$. The third set of simulations, with $a_{0}=2.0$ and $r_{0}=5 \mu \mathrm{m}$, failed to trap injected electrons since they drifted out of the wake after only a few microns of acceleration. Set one will not be discussed in greater detail, but was included for completeness.

Another effect looked at briefly was trapping due to edge effects. A sharp boundary in the simulation caused the plasma frequency to change abruptly, going from zero in vacuum to full density in a few microns. Oscillating particles will see two frequencies as they move into the vacuum and return, causing them to be dephased, and possibly trapped. This was studied previously by two other groups [23]. We chose to use the solution of Bonnaud et al. to remove this problem from the code, and moved the particle boundary accordingly. This was tested in set one, and removed almost all background trapping from the simulation. Physical boundaries this sharp are difficult to achieve experimentally.

\section{B. Simulation analysis}

Figures 8 and 9 are the longitudinal phase space $\left(p_{z} / m_{e} c\right)$ by $z$ of two different simulations. First, Fig. 8, is a pump pulse alone without injection, using the previously mentioned parameters. Electrons oscillating in the background, but not trapped in the wake, can be seen. The wake's electrostatic field is plotted also, with a strength of about $70 \%$ of wave breaking. The dashed lines represent the separatrix, calculated from the simulated wake's potential using Eq. (2). For trapping, electrons must have been moved by the injection pulse from their initial positions, to a point lying within the dashed lines. The ponderomotive drift received by the electrons from the injection pulse exceeds the predicted trapping threshold, so that many electrons should be trapped. 


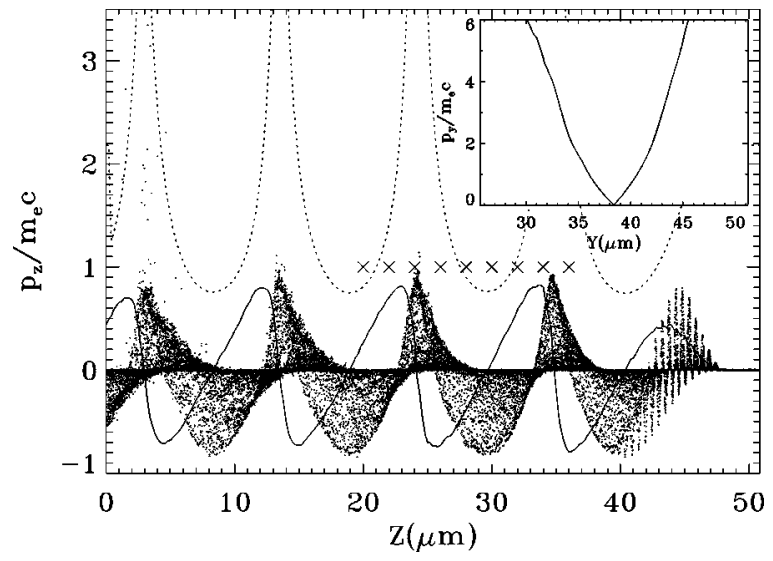

FIG. 8. LWFA only without injection. Shown are the electrons oscillating in the background (points), the normalized electrostatic field (solid line), and separatrix (dotted line). The inset shows the transverse trapping, with the maximum $p_{y} / m_{e} c=6.0$, with larger momenta drifting out sideways. The crosses mark the position where the injection pulse intersects the pump pulses' axis in each simulation of the set discussed.

Similarly to the longitudinal separatrix, one can take a transverse slice of the potential and substitute it into Eq. (5), thus yielding the maximum radial momentum for an electron to remain trapped in the wave. This is plotted in the inset of Fig. 8. To stay within the wake field, given a laser spot size of $8 \mu \mathrm{m}$ about the central axis at $38 \mu \mathrm{m}$, an electron can have at most $\left|p_{y} / m_{e} c\right| \leqslant 6.0$ on axis.

The action of the injection pulse on the wake is shown in Fig. 9. Two sets of particles appear: those in the background and those trapped in the wave. For analysis, trapping was defined by two characteristics, first if the particles had the necessary forward velocity, and secondly we artificially picked only those particles in the bucket of interest for analysis, which allowed us to calculate the properties of a single micro-bunch. In the particular simulation plotted, the injection pulse filled only buckets after it passed. The reason for

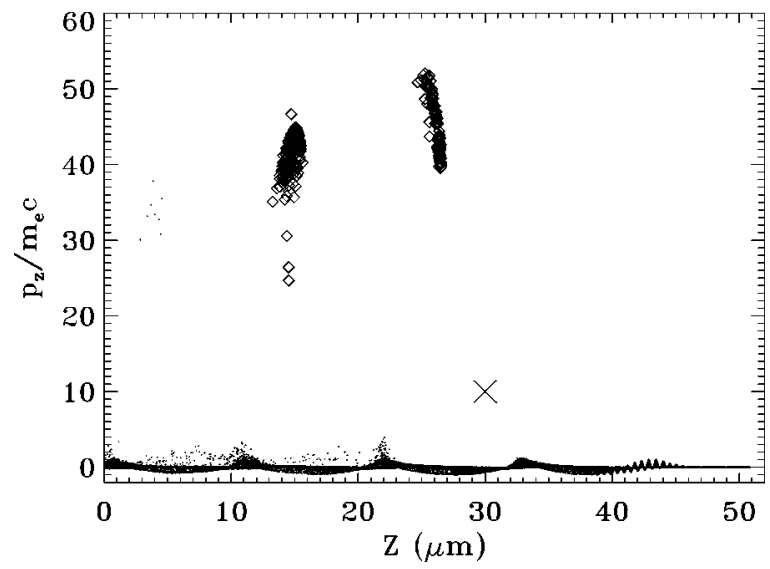

FIG. 9. LWFA with an injection pulse. Electrons trapped and accelerated in the wake may now be seen. Note that buckets after the one intended for injection are filled due to partially dephased electrons bouncing in the wake, and falling behind. Note the cross, reflecting the relative position of the injection pulse.

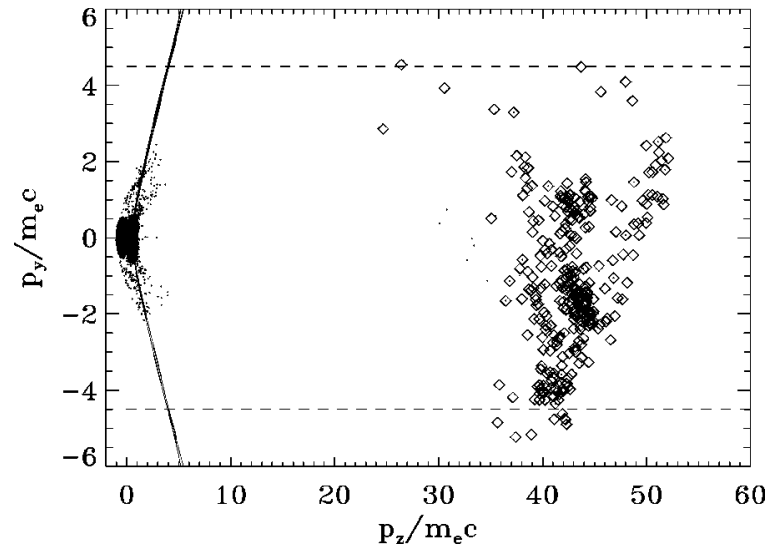

FIG. 10. This figure shows the transverse momentum $p_{y} / m_{e} c$ plotted as a function of the longitudinal momentum $p_{z} / m_{e} c$. Electrons are plotted as points, diamonds for trapped. The lines mark the region for electron trapping due to both longitudinal and transverse trapping.

this will be discussed later with individual particle trajectories. A cross marks where the peak of the injection pulse crossed the pump's axis, corresponding to a cross in Fig. 8. As the injection pulse was scanned through the different phases of the wave, electrons were injected into every bucket within the simulation domain, shown in Fig. 9. The first three buckets were analyzed for every run. A correlation between momentum and position is visible, a characteristic observed in the previous one dimensional simulations mentioned. This chirp in the bunch comes from the electrons having been injected over a finite period of time. A large area of phase space was covered as the wave advanced through different phases, subjecting the particles to remarkably different acceleration gradients. Therefore after some acceleration, the correlation between momentum and position appeared. This chirp opens the possibility of compressing the already short electron bunch by use of conventional electron-bunch compression techniques [24,25].

Motion in the transverse direction also affects trapping as previously mentioned. The injection pulse traveled in the positive y-axis direction, and kicked the electrons transversely, as well as collinear to the pump pulse axis. This was compensated by use of the transverse wake field. Plotted in Fig. 10 is the transverse momentum $p_{y} / m_{e} c$ versus $p_{z} / m_{e} c$, for the same simulation plotted previously. Around the point $(0,0)$, we see a set of points in the shape of a parabola (not the solid line), these are electrons oscillating in the background. It can be seen that the trapped particles had a much smaller transverse velocity than in the longitudinal direction, with $p_{z} / m_{e} c>10$ and $\left|p_{y} / m_{e} c\right| \leqslant 4$. Set three gave different results, longitudinally trapped particles drifted out one side of the wake field, having $p_{y}>p_{z}$. In $2 \mathrm{D}$ the separatrix changes with radius due to the wake profile, so particles within the separatrix on axis may leave it through transverse motion, but not longitudinally, even after the laser pulse has passed. Since the wake is in the two-dimensional instead of the one-dimensional limit the amplitude depends on the spot size, $r_{0}$, as well as the pump pulse's amplitude, $a_{0}$. Therefore, by making the pump pulse wider in set two, the wake field's 


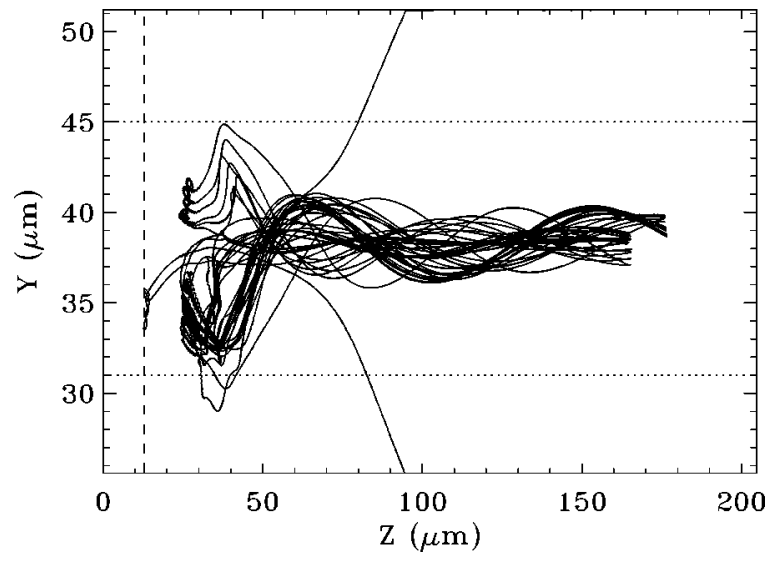

FIG. 11. Injection and acceleration process traced by 40 individual electrons in two buckets. The line with long dashes is the plasma boundary, and the dotted line shows the maximum extent of the beam oscillations in the trapping potential.

potential well deepens. Enough so that particles no longer drifted transversely out of the wake, which had decreased the number of electrons in the accelerated bunch. During trapping the electrons have a maximum radius of about $7 \mu \mathrm{m}$. The maximum transverse momentum that can be constrained to this radius is plotted with the two horizontal dashed lines in Fig. 10 at $\left|p_{y} / m_{e} c\right| \geqslant 4.5$. So the momentum of $\left|p_{y} / m_{e} c\right|$ $\geqslant 6.0$, calculated earlier using the $8 \mu \mathrm{m}$ spot size, is larger than needed to keep particles close to the axis. The parabolic solid line is the minimum longitudinal momentum from Eq. (2) at a given radius, plotted versus the maximum transverse momentum from Eq. (5) at the same radius, and depicts the absolute minimum trapping. It should be noted that the beam emittance, calculated later in this section, directly relates to the velocity spread inside the lines. We therefore conclude that particles will not drift transversely out of the wake field if the minimum focusing field is applied. The bunches were made up of electrons trapped transversely, as well as longitudinally.

Since individual particles in the bunch were identifiable, the simulation was rerun to trace trapped particles through their entire motion. This was done for 20 particles each, in the first two buckets filled in each simulation. The paths of these electrons are shown in Fig. 11, with the particles oscillating in $Y$ as they accelerate in the $Z$ direction. The oscillation's maximum extent is plotted with the dotted line, giving $r_{\max }=7.0 \mu \mathrm{m}$, previously used to find the maximum transverse velocity trapped. Figure 12 shows the longitudinal momentum versus time for two different electrons in the first two bunches of the simulation shown in Fig. 9. Figure 12(a) is an electron in the first bunch, and Fig. 12(b) in the second. The electron in Fig. 12(a) clearly oscillated in the pump pulse, and then the injection pulse superimposed on the background oscillation, after which it was trapped and accelerated. The second plot shows a similar scene, except the electron was not trapped in the first bucket, but bounced once and fell back a bucket as it moved radially through the changing separatrix. This bounce centered around a forward momentum of about $p_{z} / m_{e} c+1$, meaning the electron received a forward kick, but was still outside the separatrix for
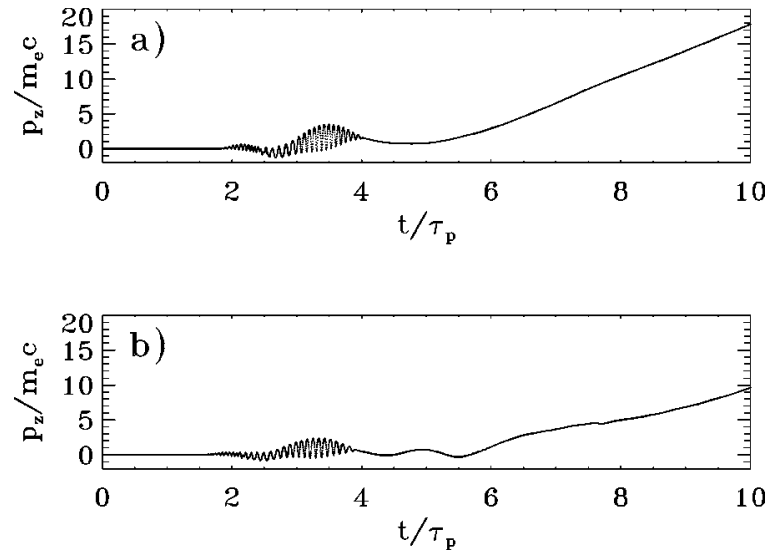

FIG. 12. Motion of a single electron. The longitudinal momentum $p_{z} / m_{e} c$ plotted as a function of time. A particle trapped in the first bucket is shown in (a), with one from the second in (b).

the first bucket. The longitudinal momentum is also plotted in Fig. 13, but now versus $z$. The dotted vertical line at $30 \mu \mathrm{m}$ is the center of the injection pulse, showing the trapped electron initially pushed in the negative $z$ direction. Figure 13(a) shows the particle from the first bucket starting near the ponderomotive force maximum at $27.5 \mu \mathrm{m}$, while the particle that slips a bucket, Fig. 13(b), comes from a region of lesser force. The orbits can actually be calculated by looking at how the ponderomotive potential effects the wave [18]. These pictures match reasonably well with Fig. 5 and Fig. 6 from the previous section, showing that all methods of analysis match each other.

Finally Figs. 14 and 15 deal with transverse motion during the trapping process. Earlier we saw particles oscillate around the pump pulse's axis due to their transverse velocity. This motion can again be seen in Figs. 14(a) and 14(b), showing the same particles as in Fig. 12, with a dashed line for the pump pulse's axis. Figure 15 diagrams the changing longitudinal trapping threshold as the particles move off axis. The solid line is the separatrix on axis from Fig. 8, while the dashed line is the separatrix at $r=7 \mu \mathrm{m}$. Off axis
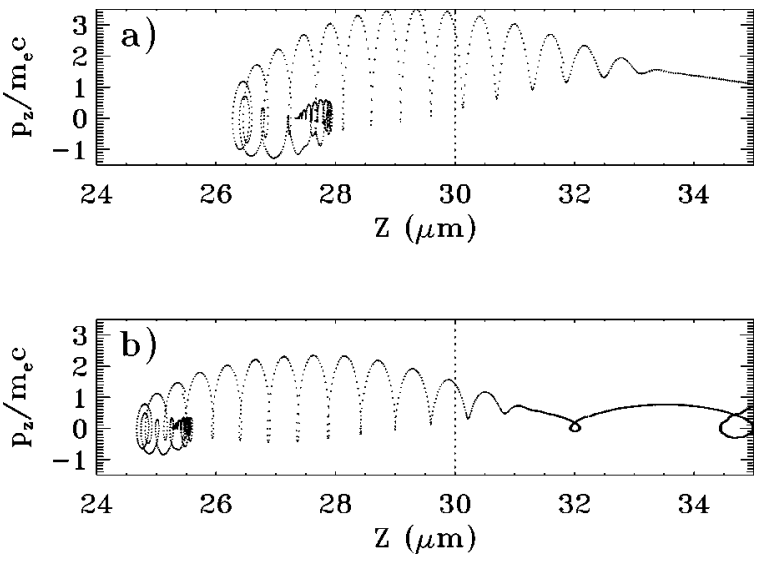

FIG. 13. The same electrons as in Fig. 12; here longitudinal momentum $p_{z} / m_{e} c$ is plotted as a function of position $Z$. (a) has the electron trapped in the first bucket with (b) showing the particle that bounces before becoming trapped. 

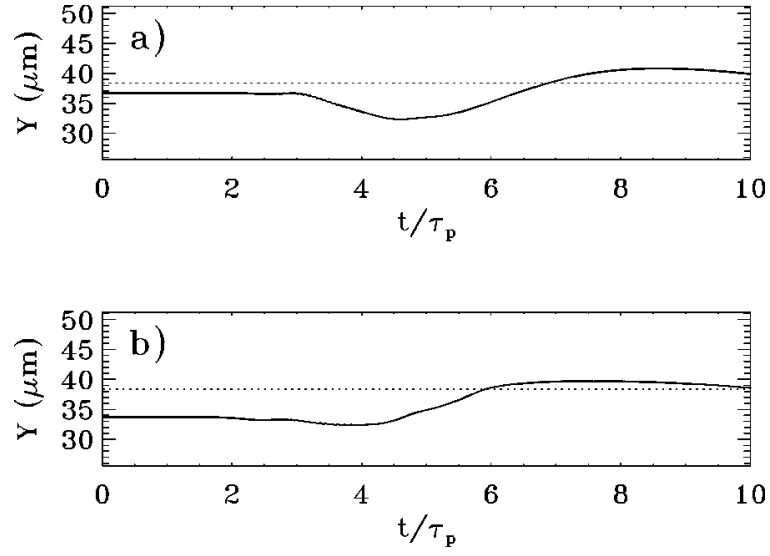

FIG. 14. The same electrons as in Fig. 12 are plotted; here transverse position $Y$ is plotted as a function of time. (a) has the electron trapped in the first bucket with (b) showing the particle that bounces before becoming trapped. The dashed line represents the axis of propagation for the pump pulse.

the minimum trapping momentum is $p_{z} / m_{e} c=2.5$ instead of 0.5 so an electron needs $1 \mathrm{MeV}$ more energy to be trapped at $r=7 \mu \mathrm{m}$ than on axis. The particles most strongly interacted with the injection pulse at a time of $t=3.7 \tau_{p}$ in both Figs. 14(a) and 14(b) were knocked off axis and then subjected to the higher trapping threshold in Fig. 15, the dashed line. Both particles can be seen to bounce back toward the center in Fig. 14, and the lower trapping threshold of the solid line in Fig. 15. However, the particle in Fig. 14(b) was overtaken by the wave and slipped back a bucket before being trapped. To be trapped, the particle's phase needed to be at the minimum of the separatrix, and also on axis at the deepest part of the well. If both criteria are not met, then no trapping occurs.

\section{Summary and comparison}

Now that it has been shown that an electron beam can be injected, it is relevant to compare the beam with existing

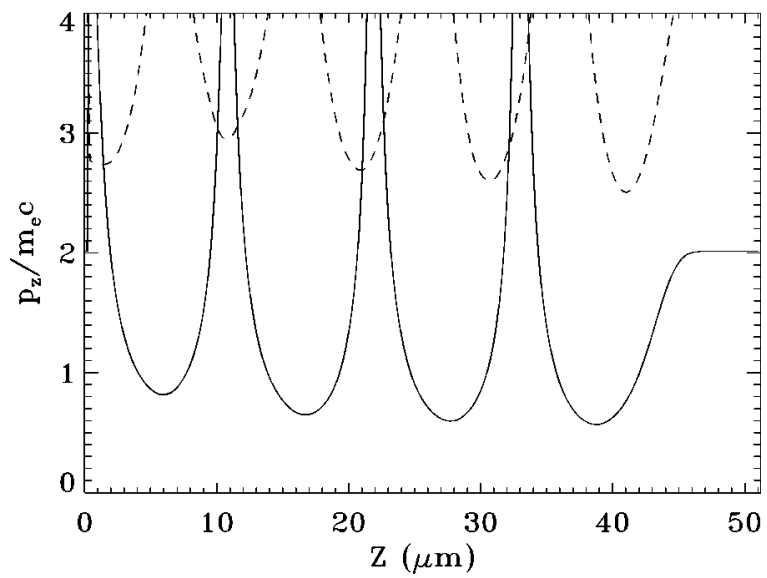

FIG. 15. The minimum trapping threshold, or separatrix. The solid line reflects the minimum momentum needed to trap an electron on axis with the pump pulse. The dashed line is the threshold for the electrons at a radius of $r_{\max }=2.5 \mu \mathrm{m}$ from the axis.
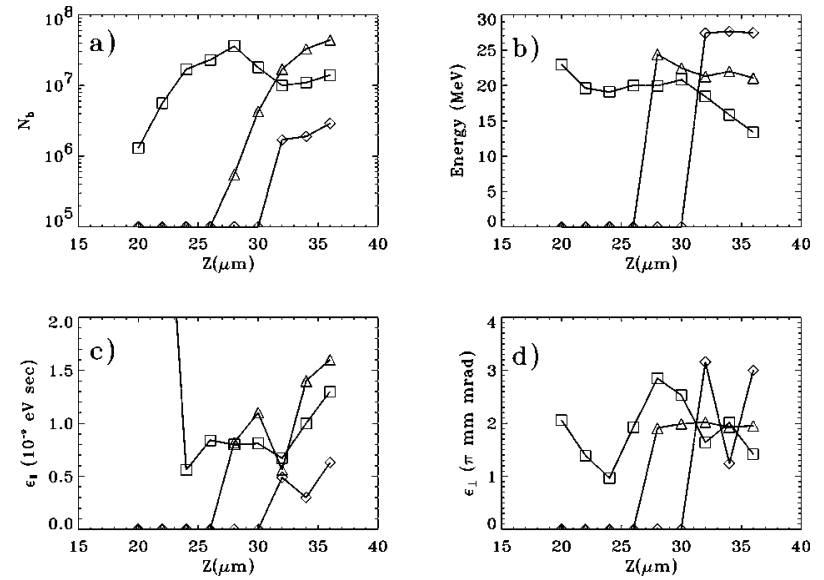

FIG. 16. Beam characteristics as a function of delay between the pump and injection pulses. Shown are (a) number of electrons in the bunch, (b) average energy of the bunch, (c) longitudinal emittance, and (d) transverse emittance. The first bucket is diamonds, second triangles, and third squares.

electron sources. First, the trapped particles mentioned previously in Sec. IV B can be converted to a number of real electrons in each bunch. Next, the bunch can be statistically analyzed for the average and standard deviation of the quantities: $z, y, p_{z}, p_{y}$. Bunch spot size and length are then $r_{0}=\sigma_{y}$ and $l=2 \sigma_{z}$, respectively. The average energy for each bunch is taken from $\overline{p_{z}}$, with an energy spread of $\sigma_{p_{z}} / \overline{p_{z}}$ and $\Delta p_{y}$ $=\sigma_{p_{y}}$ for the divergence. Figure 16 shows characteristics for the beam as a function of pulse timing, with each point corresponding to a cross in Fig. 8. To do this, we used the emittance, a common quantity used to examine a particle beam's quality, with both longitudinal and transverse emittances in two dimensions. Basically, they represent the volume of phase space occupied by the beam. In this paper, the values reported are the rms emittances, given the method of statistical analysis.

In the transverse direction, the $p_{y}-y$ plane, this volume determines the angular spread of the beam, and the spot size. In the transverse case we calculated the normalized emittance by

$$
\varepsilon_{\perp n}=\pi \gamma \beta 2 r_{0} \frac{\Delta p_{y}}{p_{z}},
$$

where $r_{0}$ is the spot size of the beam, and $p_{y}$ and $p_{z}$ are the transverse and longitudinal normalized momenta, $p / m c$. In the $2 \mathrm{D}$ simulation presented, this quantity had a value of $1-2 \pi \mathrm{mm} \mathrm{mrad}$, and was found to be constant over the simulation. The best rf guns have a value of $\sim 1 \pi \mathrm{mm}$ mrad, on the same order of magnitude [25]. 
TABLE II. Typical parameters for an electron bunch created with LILAC. The numbers for LILAC represent three microbunches clustered together.

\begin{tabular}{lccc}
\hline \hline & Photoinjectors from [25] & SMLWFA & LILAC \\
\hline Energy & $10 \mathrm{MeV}$ & $1-100 \mathrm{MeV}$ & $20 \mathrm{MeV}$ \\
Spread & $0.2-0.5 \%$ & $100 \%$ & $20 \%$ \\
Bunch length & $1-10 \mathrm{ps}$ & $\leqslant 2 \mathrm{ps}$ & $10 \mathrm{fs}$ \\
Total charge & $1-3 \mathrm{nC}$ & $1 \mathrm{nC}$ & $\leqslant 10 \mathrm{pC}$ \\
Rep. rate & $\sim 10^{2} \mathrm{~Hz}$ & $10 \mathrm{~Hz}$ & $10 \mathrm{~Hz}$ \\
Accel. field & $100 \mathrm{keV} / \mathrm{cm}$ & $2 \mathrm{GeV} / \mathrm{cm}$ & $2.3 \mathrm{GeV} / \mathrm{cm}$ \\
Accel. length & $>1 \mathrm{~m}$ & $800 \mu \mathrm{m}$ & $400 \mu \mathrm{m}$ \\
$\varepsilon_{\perp n}=\pi \gamma \beta 2 r_{0}\left(p_{y} / p_{z}\right)$ & $(1-5) \pi \mathrm{mm} \mathrm{mrad}$ & $1.1 \pi \mathrm{mm} \mathrm{mrad}$ & $\sim 2 \pi \mathrm{mm} \mathrm{mrad}$ \\
Peak current & $\sim 1 \mathrm{kA}$ & $500 \mathrm{~A}$ & $200 \mathrm{~A}$ \\
Avg. current & $\sim 100 \mathrm{nA}$ & $2 \mathrm{pA}$ & $100 \mathrm{pA}$ \\
Norm. bright. & $2 \times 10^{13} \mathrm{~A} / \mathrm{m}^{2} \mathrm{rad}^{2}$ & $4 \times 10^{13} \mathrm{~A} / \mathrm{m}^{2} \mathrm{rad}^{2}$ & $5 \times 10^{12} \mathrm{~A} / \mathrm{m}^{2} \mathrm{rad}^{2}$ \\
\hline \hline
\end{tabular}

The previous set of simulations in [10] had $\varepsilon_{\perp n}$ $=0.3 \pi \mathrm{mm}$ mrad due to smaller volumes and larger accelerating fields.

The bunch had about $3 \times 10^{7}$ electrons in it, fewer than in 1D [9]. The spot size of the electron bunch turned out to be smaller than the pump pulse due to the need to keep the electrons near the pump's axis. This change accounts for the difference between the 1D and 2D simulations. At the latest point in time of the simulation the electrons had an average energy of 20-25 MeV, and a relative energy spread of about $20 \%$. This spread in the energies is consistent with the aforementioned change in the accelerating gradient over the bunch length. If the electrons are accelerated to higher energies this value will decrease, for instance at $100 \mathrm{MeV}$ it would be only $4-5 \%$ since $\Delta E$ is roughly constant. We will represent the longitudinal emittance by the integral

$$
\varepsilon_{\|}=\oint d p_{z} d z .
$$

This quantity represents the volume of phase space filled longitudinally by the beam, representing the energy spread as well as the bunch length. It was also observed to have been a constant of the motion, with a value from the simulation of $(1-2) \times 10^{-9} \mathrm{eV} \mathrm{s}$. The longitudinal emittance is again the same or better than current devices. This is partly to do with the bunch length on the order of $3 \mu \mathrm{m}$ ( $9 \mathrm{fs}$ ). Even with a large energy spread, the area in phase space will be small with such a short bunch length. It should be noted that as the number of particles fell off when the timing between pulses was increased, the other bunch characteristics remained roughly constant. At the largest spacing between pulses the injection pulse began to overlap the plasma vacuum boundary, and once again created trapping from the boundary, therefore the last two points in Fig. 16 diverge drastically, and should be ignored.

Table II summarizes the typical characteristics in the simulations, and compares them with two other sources. One is based on a current experiment using an instability to generate electrons [8], the self-modulated laser wake-field accelerator (SMLWFA). The other comparison is a compilation of the references in [25]. Looking at these values it can be seen that LILAC compares well, with the exception of bunch number. However, the peak currents are comparable since the pulse duration is so short. The parameters for this simulation were chosen to match initial experiments, not optimal electron gun performance. If beam charges of less than 10 $\mathrm{pC}$ are undesirable, then other parameters may be explored for LILAC's pulses. Additionally there are many applications where ultrashort bunches are advantageous, such in highenergy physics, free-electron lasers, and ultrafast science. The two dimensional simulations again show that LILAC works in theory, and produces a beam of excellent quality.

\section{CONCLUSION}

Using both analysis and simulations, we have fully analyzed a concept for linear acceleration of electrons, in which a laser optically injects electrons into a wake-field plasma wave. Starting with a general analysis of the processes involved, one particular geometry was studied. The analysis was then compared to two different simulation techniques that returned similar results. In this way, a single ultrashort (9 fs) electron bunch can be trapped and accelerated up to multi-MeV energies in a millimeter distance, a particularly attractive attribute for use in many applications. By permitting femtosecond synchronization and micrometer spatial overlap between the phase of the plasma wave and the injection pulse, this technique obviates the problems associated with the alternative, attempting to combine conventional and laser-plasma accelerators. From PIC simulations we are able to conclude that this short bunch has characteristics comparable to current technology and may possibly be compressible to very short lengths. Also we have come to the conclusion that, in the case of orthogonal beams, the radial wake is a necessary part of trapping. Though this particular geometry of injection pulse may seem to create a large transverse emittance in the accelerated bunch, the predicted beam quality is competitive with photocathode rf guns under development. Parameters consistent with currently existing lasers have been used in the analysis. 


\section{ACKNOWLEDGMENTS}

We would like to acknowledge the support of the NSF and to thank Torsten Neubert and Gerard Mourou for many valuable discussions. Computing services were provided by the University of Michigan Center for Parallel Computing, which is partially funded by NSF Grant No. CDA-92-14296.
D.U. wishes to thank the Chemical Sciences, Geosciences and Biosciences Division of the Office of Basic Energy Sciences, Office of Science, U.S. Department of Energy. E.S.D. wishes to thank the U.S. Department of Energy through the University of California, Los Alamos National Laboratory under Contract No. W-7405-END-36.
[1] T. Tajima and J. M. Dawson, Phys. Rev. Lett. 43, 267 (1979).

[2] P. Sprangle et al., Appl. Phys. Lett. 53, 2146 (1988).

[3] L. M. Gorbunov and V. I. Kirsanov, Sov. Phys. JETP 66, 290 (1987); H. Hamster et al., Phys. Rev. Lett. 71, 2725 (1993); K. Nakajima et al., ibid. 74, 4428 (1995).

[4] See, e.g., Advanced Accelerator Concepts, edited by P. Schoessow, AIP Conf. Proc. No. 335 (AIP, Woodbury, NY, 1995), and references cited therein.

[5] P. Maine et al., IEEE J. Quantum Electron. 24, 398 (1988); G. Mourou and D. Umstadter, Phys. Fluids B 4, 2315 (1992); M. D. Perry and G. Mourou, Science 264, 917 (1994).

[6] E. Esarey and M. Pilloff, Phys. Plasmas 2, 1432 (1995); T. Katsouleas et al., in Laser Acceleration of Particles, edited by C. Joshi and T. C. Katsouleas, AIP Conf. Proc. 130 (AIP, New York, 1985).

[7] S. Humphries, Jr., Principles of Charged Particle Accelerators (Wiley, New York, 1986).

[8] R. Wagner et al., Phys. Rev. Lett. 78, 3125 (1997); C. I. Moore et al., ibid. 79, 3909 (1997); D. Gordon et al., ibid. 80, 2133 (1998).

[9] D. Umstadter, J. K. Kim, and E. Dodd, Phys. Rev. Lett. 76, 2073 (1996).

[10] E. Dodd, J. K. Kim, and D. Umstadter, in Advanced Accelerator Concepts, edited by S. Chattopadhyay, J. McCullough, and P. Dahl, AIP Conf. Proc. No. 398 (AIP, Woodbury, NY, 1997).

[11] A. W. Chao, R. Pitthan, T. Tajima, and D. Yeremian, Phys. Rev. ST Accel. Beams 6, 024201 (2003).
[12] B. Rau, T. Tajima, and H. Hojo, Phys. Rev. Lett. 78, 3310 (1997); E. Esarey et al., ibid. 79, 2682 (1997).

[13] S. V. Bulanov et al., Phys. Rev. Lett. 78, 4205 (1997).

[14] R. G. Hemker et al., Phys. Rev. E 57, 5920 (1998).

[15] C. Joshi et al., Nature (London) 311, 525 (1984).

[16] D. Umstadter, E. Esarey, and J. Kim, Phys. Rev. Lett. 72, 1224 (1994); D. Umstadter et al., Phys. Rev. E 51, 3484 (1995).

[17] P. Sprangle and E. Esarey, Phys. Fluids B 4, 2241 (1992).

[18] J. L. Bobin, in Advanced Accelerator Concepts (Ref. [10]).

[19] E. L. Lindman and M. A. Stroscio, Nucl. Fusion 3, 619 (1977).

[20] P. Mora and T. M. Antonsen, Phys. Plasmas 4, 217 (1997).

[21] G. G. Stokes, Trans. Cambridge Philos. Soc. 8, 441 (1847); A. D. D. Craik, Wave Interactions and Fluid Flows (Cambridge University Press, Cambridge, U.K., 1985).

[22] O. Buneman, T. Neubert, and K. Nishikawa, IEEE Trans. Plasma Sci. 20, 810 (1992); J. Villasenor and O. Buneman, Comput. Phys. Commun. 69, 306 (1992).

[23] G. Bonnaud, D. Teychenne, and J. L. Bobin, Europhys. Lett. 26, 91 (1994); S. V. Bulanov et al., Sov. J. Plasma Phys. 16, 444 (1990).

[24] M. Uesaka et al., Phys. Rev. E 50, 3068 (1994); M. Uesaka et al., Nucl. Instrum. Methods Phys. Res. A 345, 219 (1994).

[25] X. J. Wang et al., Nucl. Instrum. Methods Phys. Res. A 375, 82 (1996); C. Pellegrini et al., ibid. 341, 326 (1994); R. L. Sheffield et al., ibid. 341, 371 (1994). 